Elias Ferreira Porto ${ }^{1}$, Antonio Adolfo Matos de Castro², José Renato de Oliveira Leite ${ }^{3}$, Saul Vitoriano Miranda ${ }^{4}$, Auristela Lancauth $^{4}$, Claudia Kumpel ${ }^{5}$

1. Mestre, fisioterapêuta do Curso de Fisioterapia do Centro Universitário Adventista de São Paulo - UNASP, São Paulo (SP), Brasil e do Grupo de Pesquisa sobre Membros Superiores e Hiperinsuflaçáo Pulmonar em DPOC da Universidade Federal de São Paulo UNIFESP, São Paulo (SP), Brasil. 2. Mestre, Professor do Curso de PósGraduaçáo em Terapia Intensiva do Centro Universitário Adventista de São Paulo UNASP, Fisioterapeuta do Instituto do Coraçáo (InCor) do Hospital das Clínicas da Faculdade de Medicina da Universidade de São Paulo - USP, São Paulo (SP), Brasil; Pesquisador do Centro de Reabilitaçáo Pulmonar da Universidade Federal de São Paulo - UNIFESP, São Paulo (SP), Brasil. 3. Professor do Curso de Terapia Intensiva do Centro Universitário Adventista de São Paulo - UNASP - São Paulo (SP), Brasil e Fisioterapeuta do Curso de Fisioterapia Cardiorrespiratória do Instituto do Coração (InCor) do Hospital das Clínicas da Faculdade de Medicina da Universidade de São Paulo - USP, São Paulo (SP), Brasil; 4. Aluno da Especialização em Fisioterapia em Terapia Intensiva do Centro Universitário Adventista de São Paulo UNASP, São Paulo (SP), Brasil. 5. Mestre, Professora do Curso de Fisioterapia do Centro Universitário Adventista de São Paulo - UNASP, São Paulo (SP), Brasil.

Recebido da Universidade Federal do Estado de São Paulo - UNIFESP, São Paulo (SP), Brasil.

Submetido em 20 de fevereiro de 2008 Aceito em 06 de agosto de 2008

Endereço para correspondência: Elias Ferreira Porto

R. Almirante Luiz Penido Burnier 47/24 Jardim Sandra

05860-000 São Paulo, SP

E-mail: eliasfporto@gmail.com

\section{Análise comparativa da complacência do sistema respiratório em três diferentes posiçóes no leito (lateral, sentada e dorsal) em pacientes submetidos à ventilaçáo mecânica invasiva prolongada}

\author{
Comparative analysis of respiratory systems compliance in \\ three different positioning (lateral, dorsal and sitting) in \\ patients in prolonged invasive mechanical ventilation
}

\section{RESUMO}

Objetivos: A realização deste estudo se justifica pelo fato que na prática clinica ocorrem constantes mudanças de decúbito do paciente no leito durante a hospitalizaçáo na terapia intensiva, sendo que necessita melhor entendimento sobre possíveis efeitos adversos principalmente sobre as condiçóes do sistema respiratório que tais mudanças podem ocasionar. $\mathrm{O}$ objetivo deste estudo foi avaliar se o posicionamento do paciente no leito pode interferir na complacência pulmonar.

Métodos: Todos os pacientes incluídos neste estudo estavam em ventilação mecânica, e foram sedados e curarizados. Verificou-se a complacência do sistema respiratório de todos os pacientes em três diferentes posicionamentos: decúbito lateral (DL), decúbito dorsal (DD) e sentado (PS), para tanto, após a manobra de recrutamento alveolar os pacientes ficavam no posicionamento definido por 2 horas e nos últimos $5 \mathrm{~min}$ os dados eram colhidos do mostrador do ventilador mecânico.
Resultados: Vinte e oito pacientes foram prospectivamente analisados, respiratório no DL foram $37,07 \pm 12,9$ no DD 39,2 $\pm 10,5$ e na PS $43,4 \pm 9,6$ $\mathrm{mL} / \mathrm{cmH}_{2} \mathrm{O}$. Houve diferença estatisticamente significativa quando a PS e a DD foram comparadas com a DL para complacência o sistema respiratório $(\mathrm{p}=$ 0.0052) e volume corrente ( $<<0.001)$. Houve correlação negativa entre os valores médios de pressão expiratória final positiva e complacência do sistema respiratório $(r=0,59, p=0,002)$. Para o $\mathrm{DL}$ a $\mathrm{FIO}_{2}$ foi 0,6 , para o DD e posição sentada foi $0,5 .(p=0,049)$.

Conclusóes: $\mathrm{O}$ posicionamento dos pacientes no leito, em ventilação mecânica invasiva, ocasiona variaçáo na complacência do sistema respiratório, volume corrente e saturaçáo arterial de oxigênio. $\mathrm{Na}$ posiçáo sentada a complacência do sistema respiratório é maior quando comparada aos decúbitos dorsal e lateral.

Descritores: Complacência pulmonar; Respiração artificial Os valores de complacência do sistema

\section{INTRODUÇÁO}

Um aspecto importante a ser avaliado antes do desmame ventilatório é a complacência do sistema respiratório, pois desmame da ventilação mecânica pode ser prejudicado pela deficiente condição pulmonar do paciente. ${ }^{1,2}$ A complacência do sistema respiratório é definida como a inclinação da curva de pressão-volume, ou a variação de volume por unidade de alteração de pressão. Os pulmôes e o tórax são constituídos por tecidos com propriedades elásticas, portanto, a complacência do sistema respiratório é uma medida de sua elasticidade e de sua resistência à deformidade frente a qualquer força representada por graus variáveis de esforço. ${ }^{3}$

A complacência do sistema respiratório pode ser medida com o paciente em ventilação mecânica e sob efeito de sedaçãó ${ }^{4}$, sendo o seu cálculo expresso pela di- 
visão do volume-corrente pela pressão de pico menos a pressão positiva ao final da expiraçáo (PEEP). Para a realizaçáo da medida de complacência do sistema respiratório com o paciente em ventilação mecânica é recomendado realizar recrutamento alveolar prévio, no intuito de homogeneização de todas as áreas pulmonares. ${ }^{3}$

Um dos fatores que podem interferir reduzindo ou aumentando a complacência do sistema respiratório é o posicionamento do paciente no leito. ${ }^{4}$ Atualmente se preconiza que ocorram mudanças de posicionamento a cada duas horas nos pacientes internados em unidade de terapia intensiva (UTI). ${ }^{5}$

Estudos mostram que ocorrem alteraçóes significativas da complacência do sistema respiratório entre as posições sentado e decúbito lateral, bem como aumento do pico de pressão inspiratória. Posteriormente, este mesmo grupo mostrou que ocorrem alteraçóes na complacência do sistema respiratório, com o prolongamento do paciente em ventilação mecânica, sendo este um fator que aumentou o risco para re-intubaçáo de paciente. ${ }^{1,6}$

O objetivo deste estudo foi avaliar o posicionamento do paciente no leito hospitalar que proporciona melhor complacência pulmonar em pacientes sob ventilação mecânica invasiva, visando sucesso no desmame ventilatório.

\section{MÉTODOS}

Após aprovação pela Comissão de Ensino e Pesquisa dos hospitais, foi realizado o estudo em dois Hospitais da rede privada da cidade de São Paulo, no período de fevereiro a dezembro de 2006.

Foram incluídos os pacientes hospitalizados em UTI submetidos à ventilaçáo mecânica prolongada; com idade entre 18 e 81 anos, hemodinamicamente estáveis e sem diagnóstico prévio de síndrome do desconforto respiratório agudo (SDRA) e fibrose pulmonar.

Os dados coletados foram: idade, diagnóstico clínico do paciente, parâmetros ventilatório e hemodinâmico. Os dados ventilatórios foram colhidos por meio do mostrador do ventilador mecânico Raphel da Hamilton Medical.

Solicitou-se à equipe médica que promovesse a sedação e analgesia no intuito de inibir estímulos respiratórios dos pacientes. Todos os pacientes estavam sendo ventilados na modalidade controlada a pressão (CMP), no qual todos os ciclos ventilatórios eram fornecidos e controlados pelo ventilador mecânico; portanto, não permitindo mecanismo alternativo de ciclagem.

A coleta foi realizada em três posiçôes: decúbito lateral (DL), posição sentada (PS) e decúbito dorsal (DS). A ordem de início para o posicionamento do paciente foi aleatória.

Após a aleatorização o paciente era posicionado no leito de forma confortável, sendo que após duas horas que o mesmo estava posicionado era realizada homogeneização pulmonar com fração inspirada de oxigênio $\left(\mathrm{FiO}_{2}\right)$ a $100 \%$ mediante a elevação da PEEP de $2 \mathrm{em} 2 \mathrm{cmH}_{2} \mathrm{O}$ até alcançar uma PEEP de $20 \mathrm{cmH}_{2} \mathrm{O}$, mantendo-a por 2 minutos. Em seguida, a PEEP era reduzida de $2 \mathrm{em} 2 \mathrm{cmH}_{2} \mathrm{O}$ até o nível de PEEP inicial. Após a homogeneização os dados eram coletados. Os pacientes permaneciam no posicionamento preconizado por mais 5 min com $\mathrm{FiO}_{2}$ mínima para manter saturação arterial de oxigênio $\left(\mathrm{SaO}_{2}\right)$ maior do que $93 \%$. Após duas horas no posicionamento seguinte, realização da homogeneização pulmonar e coleta dos dados a $\mathrm{SaO}_{2}$ deveria manter a mesma, mas se o paciente apresentasse dessaturação maior do que 3\% ou $\mathrm{SaO}_{2}$ menor do que $90 \%$ a $\mathrm{FiO}_{2}$ seria aumentada.

Foram coletados os valores de volume-corrente, pico de pressão inspiratória, fluxo inspiratório, volume-minuto, freqüência respiratória, pressão de platô inspiratório, pressão positiva final da expiração e fluxo inspiratório, pressão média de vias aéreas, relação espaço-morto/volume-corrente obtidos diretamente do display do respirador. Esses valores eram monitorados respiração a respiração por 1 minuto. Para a avaliaçáo da mecânica respiratória retirou-se o umidificador do circuito do respirador e realizaram-se as medidas de complacência estática do sistema respiratório $\left(\mathrm{mL} / \mathrm{cmH}_{2} \mathrm{O}\right)$, resistência das vias aéreas $\left(\mathrm{cmH}_{2} \mathrm{O} / \mathrm{L} / \mathrm{m}\right)$, relaçãoo espaço-morto/volume- corrente.

Obteve-se a complacência estática dividindo-se o volumecorrente pela pressão de platô subtraída do valor da PEEP. Calculou-se a resistência das vias aéreas total dividindo-se a diferença entre o pico de pressão inspiratória e a pressão platô pelo fluxo inspiratório.

O software do ventilador mecânico possibilitava o cálculo indireto dos dados da pressão pleural e alveolar. Esses dados eram monitorados respiração a respiraçáo por meio do mostrador do ventilador mecânico. A pressão transpulmonar foi calculada pela subtraçáo da pressão alveolar pela pressáo pleural. A complacência dinâmica do sistema respiratório era mensurada pela fórmula: volume-corrente/pressão de pico inspiratório subtraída do valor da PEEP. ${ }^{2}$ A freqüência respiratória, a PEEP e a fração inspirada de oxigênio eram mantidas durante todo o procedimento. Para a análise estatística foi considerado o maior valor obtido de cada um desses parâmetros.

Após a coleta dos dados, o paciente era posicionado no decúbito seguinte determinado pelo sorteio e os mesmos procedimentos eram repetidos.

\section{Análise Estatística}

Os dados estão expressos em média \pm desvio padrão, mínimo e máximo. A análise das variáveis em três momentos foi feita por meio da análise de variância de medidas repetidas (ANOVA RM), com pós-teste de Bonferroni. Para analisar a 
força associativa entre as variáveis utilizou-se a correlação de Pearson. Foi considerado como valor estatisticamente significativo $\mathrm{p}<0,05$.

\section{RESULTADOS}

Fizeram parte deste estudo 28 pacientes, sendo 17 do sexo masculino, com a média da idade de $51,1 \pm 17,4$ anos (18 e 81). O diagnóstico mais freqüente foi neoplasia intestinal que ocorreu em $28,5 \%$ dos pacientes; a complicação mais freqüente foi a insuficiência respiratória que ocorreu em $60,7 \%$ dos pacientes, não houve nenhuma intercorrência durante os procedimentos de coleta dos dados, apenas cinco pacientes apresentaram $\mathrm{SpO}_{2}$ abaixo de $90 \%$ em decúbito lateral, e um em decúbito dorsal.

Todos os pacientes do estudo estavam em ventilação mecânica invasiva na modalidade controlada e a pressão (CMP).
Os dados estão apresentados no quadro 1

Os valores médios de complacência do sistema respiratório em DL foram 37,07 $\pm 12,9 \mathrm{~mL} / \mathrm{cmH}_{2} \mathrm{O}$, em DD $39,2 \pm 10,5 \mathrm{~mL} / \mathrm{cmH}_{2} \mathrm{O}$ e em PS 43,4 $\pm 9,6 \mathrm{~mL} / \mathrm{cmH}_{2} \mathrm{O}$, respectivamente. Houve diferença estatisticamente significativa nos valores médios de complacência do sistema respiratório quando comparados à posição sentada e o decúbito dorsal com o decúbito lateral $(\mathrm{p}=0,0052)$ (Figura 1). Para a complacência pulmonar não encontramos diferença estatisticamente significante para os três posicionamentos (Figura 2)

Os valores médios do volume-corrente exalado no DL foi $670 \pm 202 \mathrm{~mL}$, no DD foi $690 \pm 229 \mathrm{~mL}$ e na PS $705 \pm 269 \mathrm{~mL}$. Houve diferença estatisticamente significativa entre o DD e a PS, em relação ao DL $(\mathrm{p}<0,001)$ (Figura 3).

A média da pressão de pico no DL foi 24,6 \pm 4,5 (14

Quadro 1 - Características demográficas, diagnóstico e complicaçóes clínicas nos pacientes estudados $(\mathbf{n}=28)$

\begin{tabular}{|c|c|c|c|}
\hline Sexo & Idade (anos) & Diagnóstico & Complicações \\
\hline M & 59 & AVE isquêmico + insuficiência hepática & Pneumonia, IrpA \\
\hline $\mathrm{F}$ & 61 & Neoplasia de colon, aplasia Medular & Choque séptico, IrpA \\
\hline $\mathrm{F}$ & 59 & Neoplasia de endométrio & Choque Séptico \\
\hline M & 74 & Edema agudo de pulmão, ICC & IRA, choque, IrpA, pneumonia, \\
\hline M & 64 & ICC & Derrame pleural \\
\hline M & 65 & Cardiomegalia, chagas & IrpA, sepse \\
\hline $\mathrm{F}$ & 26 & Traumatismo cranioencefálico, politrauma & Hemotórax, IrpA \\
\hline M & 74 & Cetoacidose diabética & Sepse \\
\hline M & 22 & Traumatismo cranioencefálico & Pneumonia \\
\hline M & 47 & Politrauma & Choque séptico \\
\hline $\mathrm{F}$ & 30 & Câncer de intestino & Derrame pleural \\
\hline $\mathrm{F}$ & 18 & Lesão medular torácica alta & Pneumonia \\
\hline M & 53 & Infarto agudo do miocárdio & Choque cardiogênico \\
\hline $\mathrm{F}$ & 60 & Insuficiência renal & Edema pulmonar \\
\hline $\mathrm{F}$ & 31 & Sepse & Choque séptico \\
\hline M & 70 & Choque cardiogênico & $\operatorname{IrpA}$ \\
\hline M & 55 & Traumatismo cranioencefálico & Pneumotórax \\
\hline M & 81 & Neoplasia de pulmão & $\operatorname{IrpA}$ \\
\hline M & 52 & Ressecção de reto por neoplasia & Nenhuma \\
\hline M & 41 & Tromboembolismo pulmonar & $\operatorname{IrpA}$ \\
\hline M & 61 & Coledocoplastia por neoplasia & Sepse \\
\hline M & 69 & Retosigmoidectomia & Nenhuma \\
\hline M & 63 & Infarto agudo do miocárdio ântero-lateral & Choque cardiogênico \\
\hline M & 45 & Insuficiência renal, neoplasia metastática no pulmão esquerdo & $\operatorname{IrpA}$ \\
\hline $\mathrm{F}$ & 35 & Sepse & Choque séptico \\
\hline $\mathrm{F}$ & 30 & Insuficiência respiratória & Nenhuma \\
\hline $\mathrm{F}$ & 31 & Insuficiência respiratória & Nenhuma \\
\hline $\mathrm{F}$ & 55 & Insuficiência respiratória & Nenhuma \\
\hline
\end{tabular}

AVE - acidente vascular encefálico, ICC - insuficiência cardíaca congestiva, IRA - insuficiência renal aguda, IrpA - insuficiência respiratória 


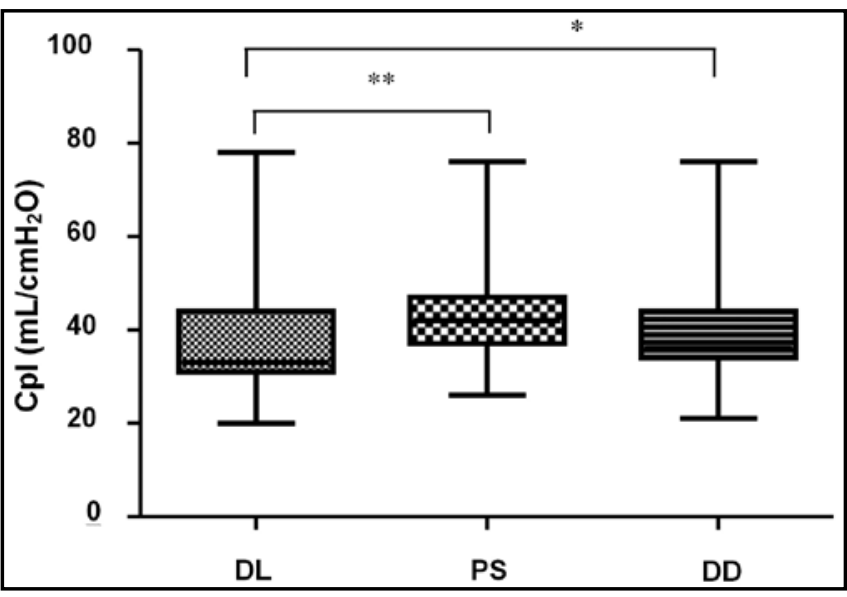

Figura 1 - Valores de complacência do sistema respiratório nos três posicionamentos estudados. $\mathrm{Clp}$ - complacência, DL - decúbito lateral, PS - posição sentada, DD - decúbito dorsal. * $\mathrm{p}<0,01,{ }^{* *} \mathrm{p}<0,001$

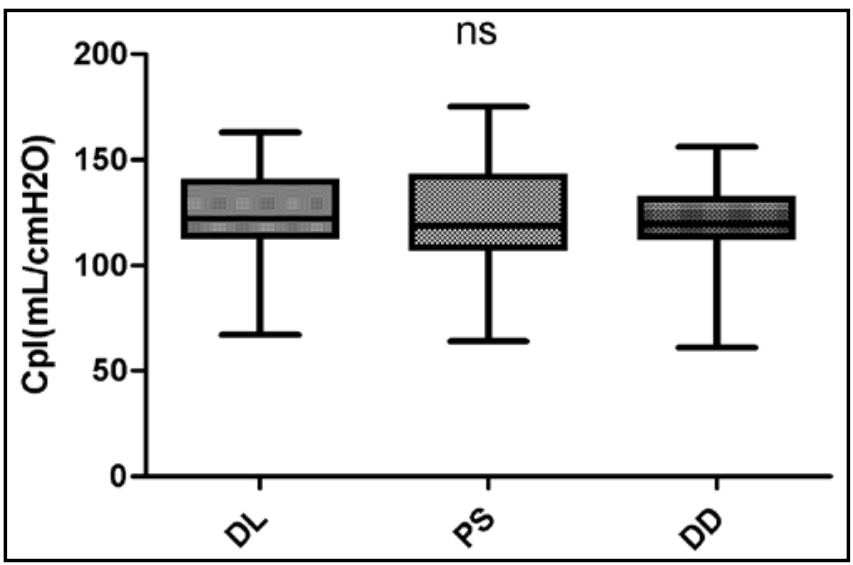

Figura 2 - Valores de complacência pulmonar nos três posicionamentos estudados. Clp - complacência, DL - decúbito lateral, PS - posição sentada, DD - decúbito dorsal, NS - não significativo

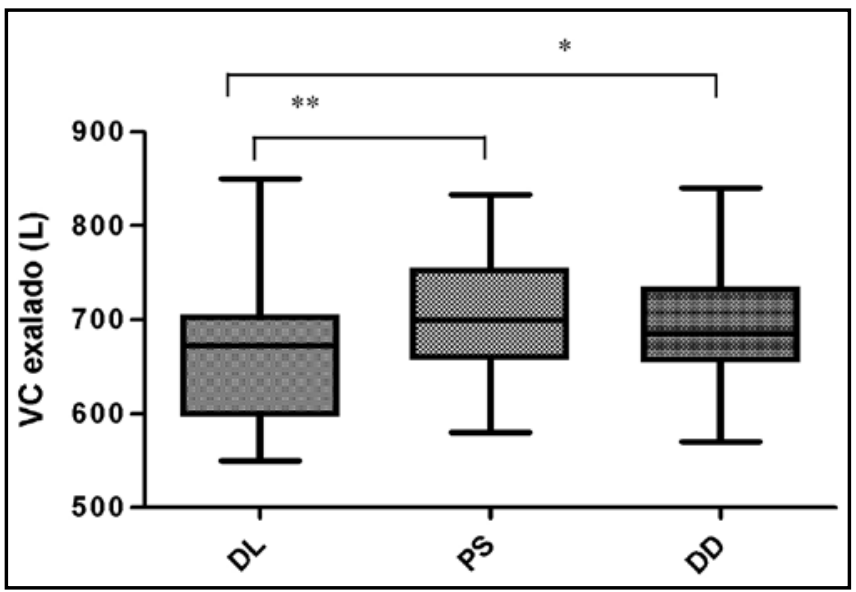

Figura 3 - Valores de volume corrente nos posicionamentos estudados. VC - volume corrente, DL - decúbito lateral, PS - posição sentada, DD - decúbito dorsal. * $\mathrm{p}<0,01,{ }^{* *} \mathrm{p}<0,001$ a 31); no DD a média foi $24,6 \pm 4,7$ (14 a 30). Já na PS a média foi $25,3 \pm 4,7$ (16 a 35). Não havendo, portanto, diferença das pressões de pico nos diferentes posicionamentos $(\mathrm{p}=0,81)$.

A pressão alveolar foi significativamente maior na PS $\left(21 \mathrm{cmH}_{2} \mathrm{O}\right)$ em relaçáo DL $\left(18 \mathrm{cmH}_{2} \mathrm{O}\right) \mathrm{p}=0,0067$, mas não foi encontrada diferença entre o DD, PS e DL. A pressão transpulmonar foi maior na PS $(\mathrm{p}<0,001)$ e DD $(\mathrm{p}<0,01)$ do que o DL (Figura 4)

Os valores médios da pressão de vias aéreas nos DL e $\mathrm{DD}$, respectivamente, foi $15,9 \pm 5,7$ e $16,4 \pm 6,6$; e na PS foi de $16,9 \pm 7,3$, não havendo diferença estatística entre si $(p=0,34)$. Os valores médios de PEEP nos três posicionamentos foram semelhantes, não havendo diferença estatística $(p=0,99)$. Houve correlação negativa entre os valores médios de PEEP e complacência pulmonar $(r=$ $-0,59, \mathrm{p}=0,002)$.

Os valores médios da freqüência respiratória nos três posicionamentos foram semelhantes, não havendo diferença estatística significativa $(\mathrm{p}=0,99)$.

Os valores médios do fluxo inspiratório no DL e DS foram $65,8 \pm 21,3$ e $63 \pm 19 \mathrm{~L} / \mathrm{s}$, respectivamente; na PS foi $65,7 \pm 6,8 \mathrm{~L} / \mathrm{s}$. Houve diferença estatisticamente significativa quando comparado o DL com as demais posiçóes $(\mathrm{p}=0,044)$.

Houve diferença estatística significativa $(\mathrm{p}=0,049)$ em relação aos valores médios da fração inspirada de oxigênio ofertada nos posicionamentos sentado e dorsal em relação ao DL, visto que a $\mathrm{FIO}_{2}$ seria oferecida minimamente suficiente para manter a $\mathrm{SpO}_{2}$ maior ou igual a $93 \%$. Para o $\mathrm{DL}$ a $\mathrm{FIO}_{2}$ foi 0,6 ; para o DS e PS foi 0,5 .

A figura 5 mostra os resultados obtidos nos três posicionamentos em relação aos valores médios do volume-

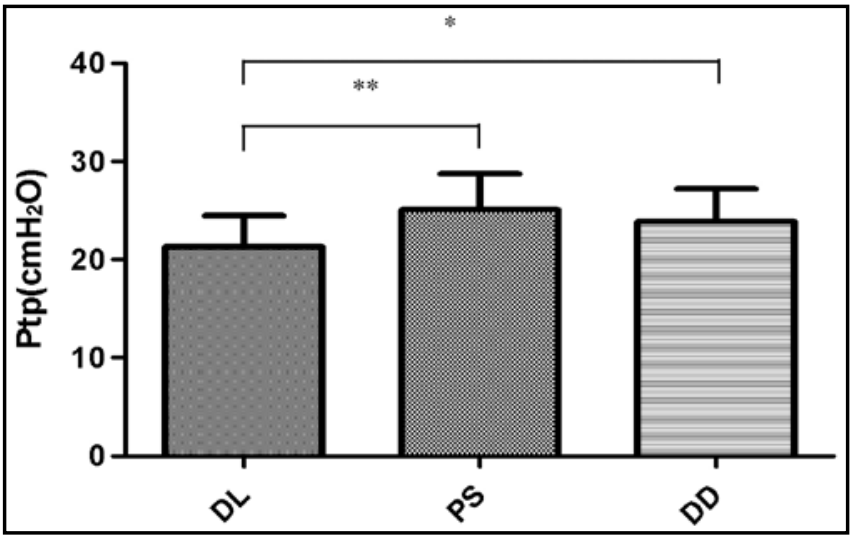

Figura 4 - Valores médios de pressáo transpulmonar nos posicionamentos estudados. Ptp - pressão transpulmonar, DL decúbito lateral, PS - posição sentada, DD - decúbito dorsal. * $\mathrm{p}<0,01,{ }^{* *} \mathrm{p}<0,001$ 
minuto. Verificou-se que o volume-minuto foi menor no decúbito lateral quando comparado com decúbito dorsal a posição sentada $(\mathrm{p}<0,0002)$.

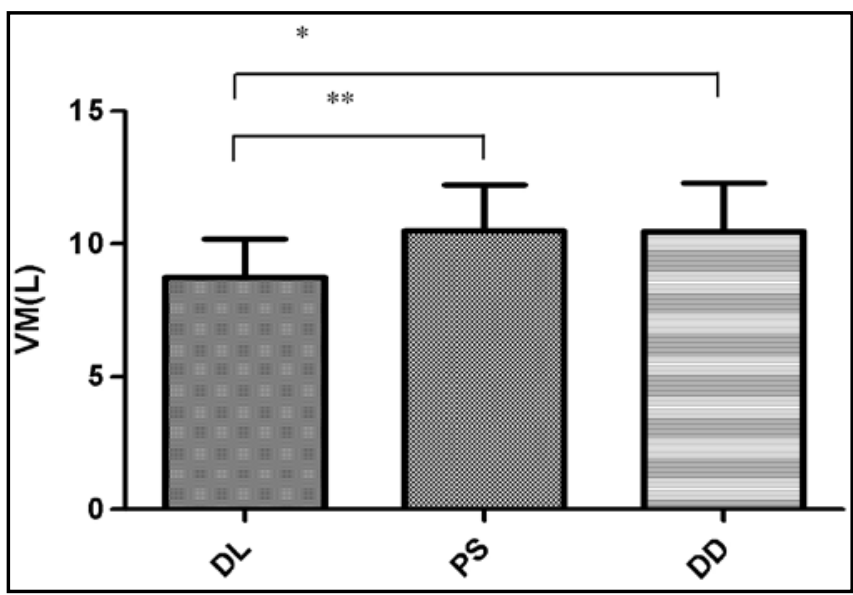

Figura 5 - Valores médios de ventilaçáo minuto nos posicionamentos estudados. VM - volume minuto, DL - decúbito lateral, PS - posição sentada, DD - decúbito dorsal. * $\mathrm{p}<0,01$, ** $\mathrm{p}<0,001$

\section{DISCUSSÃO}

Prioritariamente, o presente estudo analisou a influência do posicionamento corporal sobre as alteraçóes fisiológicas de pressóes, volumes e fluxos pulmonares. O principal achado é o fato de a posição sentada ter proporcionado o maior valor de complacência do sistema respiratório, embora náo foi encontrado o mesmo para complacência pulmonar, maior volume-corrente e, conseqüentemente volume-minuto; e inversamente ter apresentado menor fluxo inspiratório pulmonar.

Alguns autores relatam que em condiçóes normais as regióes inferiores dos pulmóes ventilam melhor do que as superiores. ${ }^{7,8}$ Porém foi observado que em pacientes no DL durante a ventilaçáo mecânica controlada, o pulmão contra-lateral foi o que melhor ventilou já que suas unidades alveolares são mais distensíveis e com menor resistência ao fluxo aéreo do que o pulmão homolateral (zona pulmonar dependente).?

As funçōes pulmonares podem ser afetadas pelas mudanças de posicionamentos ${ }^{7}$. A importância da mudança de posição em determinados períodos ( $2 \mathrm{em} 2$ horas) se dá não apenas para evitar lesôes dermatológicas, mas, também melhorar as funçóes respiratória e vascular. ${ }^{10}$

No presente estudo foi observado que o posicionamento do paciente no leito interfere significativamente na complacência do sistema respiratório assim como o nível de PEEP a ser utilizada. Observou-se que quanto maior era o valor da PEEP utilizada, pior a complacência pulmonar encontrada. Já que, a PEEP promove oxigenação por meio do efeito de aumento da área seccional transversa do alvéolo, o que garante um estado de hiperinsuflação pulmonar. Este estado possibilita pouco aumento de volume pela pressão gerada pelos músculos da respiraçáo, ou seja, pouco aumento de complacência pulmonar; ao contrário, o maior vetor de força gerado é a de recuo elástico, isto é, de maior elastância.

Alguns estudos mostraram maior complacência do sistema respiratório no decúbito lateral, e verificaram que nessa posição o peso do mediastino e o deslocamento do conteúdo abdominal contribuíram para o gradiente de pressáo pleural vertical ${ }^{11-13}$ e sugeriram, portanto que, apenas o DL proporciona maior complacência do sistema respiratório. Em contrapartida, outro estudo demonstrou que a complacência do sistema respiratório é maior nas PS e DD, quando comparadas ao DL; possivelmente seja pelo fato de que as unidades alveolares são menos distensíveis e oferece maior resistência ao fluxo aéreo pela desvantagem mecânica apresentada pelo paciente quando em DL.?

No presente estudo observou-se maior complacência do sistema respiratório na posição sentada em relação aos decúbitos lateral e dorsal $(\mathrm{p}=0,0052)$. Atribui-se esse fato à zona dependente na posição sentada, que é a área da base pulmonar. Efetivamente, esta possui menor tamanho que as áreas das regióes dependentes nos outros posicionamentos. Sendo assim permite que o restante das zonas não dependentes estejam mais complacentes. Outros fatores que pode ser alterado com a mudança de posicionamento são as constantes de tempo ocasionando desigualdade na distribuição espacial da ventilação, as unidades de menor complacência enchem mais rápido que as demais. Unidades pulmonares com elevada complacência e resistência de vias aéreas normal têm uma grande expansão, enquanto que aqueles com baixa complacência têm menor tempo de enchimento. ${ }^{14}$

Foi observado também que o volume-corrente foi maior na posição sentada do que nos decúbitos dorsal e lateral $(p=0,001)$. Relacionou-se este fato com a complacência do sistema respiratório, a qual é uma medida de elasticidade e resistência, à deformidade frente a qualquer força representada por graus variados de esforço. ${ }^{15,16}$ Foi verificado também que o fluxo pulmonar é menor no decúbito dorsal do que no lateral e na posiçáo sentada ( $\mathrm{p}=$ $0,044)$. Isto provavelmente se deve ao fato de haver maior compressão mecânica na caixa torácica no decúbito dorsal, diminuindo, portanto, o fluxo respiratório.

Em relação específica à posição sentada, o fluxo inspiratório foi menor, provavelmente esse fato ocorreu pelo 
aumento da resistência de via aérea. Essa mesma posição foi a que garantiu maior complacência do sistema respiratório quando comparada aos outros posicionamentos e consecutivamente maior ventilação pulmonar. De forma geral isto pode ter ocorrido, pela grande distensibilidade ocorrida pela alta quantidade de ar, preenchendo os alvéolos e ocasionando a diminuição do fluxo inspiratório, ou seja, próximo ao máximo da capacitância alveolar. Conseqüentemente, há um aumento exponencial na resistência de via aérea e no parênquima pulmonar. A resistência é expressa pela quantidade de pressão intratorácica produzida dividida pelo fluxo pulmonar. Portanto, nesta condição seria necessário um grande aumento de pressão muscular para gerar um mínimo de fluxo inspiratório, devido à grande distensibilidade e resistência alveolar. ${ }^{17,18}$

As limitaçóes deste estudo se deram pelo fato de não se ter colhido os dados de forma continua por duas horas como sugerem os estudos, que as mudanças de decúbito devem ser feitas a cada duas horas, assim se poderia ter mais bem quantificadas as alteraçóes da complacência pulmonar nos diferentes posicionamentos, assim como a obtenção da pressão pleural e alveolar por um método indireto, o qual seria mais confiável se obtida por meio da passagem do balão esofágico.

\section{CONCLUSÓES}

Concluiu-se que o posicionamento corporal de pacientes em ventilação mecânica invasiva ocasiona variação na complacência do sistema respiratório. Na posição sentada a complacência do sistema respiratório é maior quando comparada aos decúbitos dorsal e lateral.

Na posição sentada ocorre menor fluxo inspiratório do que nos decúbitos dorsal e lateral. $\mathrm{E}$, independentemente do posicionamento corporal há diminuição progressiva da complacência pulmonar à medida que ocorre aumento progressivo de pressão positiva no final da expiração. Estas conclusóes nos levam a sugerir que ao realizarem mudanças de posicionamento do paciente no leito deve ser cuidadosamente avaliada as alteraçóes da mecânica ventilatória do paciente.

\section{ABSTRACT}

Objectives: This study is justified by the fact that in clinical practice, changes occur in patient's positioning in the bed during hospitalization in intensive care unity, it's necessary better understanding about possible adverse effects that such changes might cause mainly on the respiratory system condition. The objective this study was to evaluate if the patients positioning in bed can to alter the pulmonary complacency.

Methods: All included patients were submitted to mechanical ventilation and were sedated and curarized respiratory system compliance was assessed in three different positioning: lateral, dorsal and sitting. After an alveolar recruitment maneuver, patients were placed to a position throughout two hours, and in the last five minutes the data was collected from the mechanical ventilator display.

Results: twenty eight patients were prospectively assessed. Values of respiratory system compliance in the lateral position were $37,07 \pm 12,9$ in the dorsal were $39,2 \pm 10,5$ and in the sitting 43,4 $\pm 9,6 \mathrm{~mL} / \mathrm{cmH}^{2} \mathrm{O}$. There were a statistical difference when we compared to the sitting and dorsal with lateral positioning for respiratory system compliance $(\mathrm{p}=0.0052)$ and tidal volume $(\mathrm{p}<0.001)$. There was a negative correlation between mean values of positive end expiratory pressure a respiratory system compliance $(\mathrm{r}=0.59, \mathrm{p}=0.002)$. The $\mathrm{FIO}_{2}$ administered was 0.6 for the lateral positioning and 0.5 for the dorsal and sitting positioning $(\mathrm{p}=0.049)$.

Conclusions: That body positioning in patients restrained to a bed and submitted to invasive mechanical ventilation leads to pulmonary compliance, tidal volume and $\mathrm{SpO}_{2}$ oscillations. In the sitting position the pulmonary compliance is higher than in others positions.

Keywords: Pulmonary compliance, Artificial respiration

\section{REFERÊNCIAS}

01. Meade M, Guyatt G, Cook D et al - Predicting success in weaning from mechanical ventilation. Chest, 2001;120:(Suppl6):400S-424S.

02. Azevedo JRA. Ventilação mecânica prolongada e desmame do respirador na insuficiência respiratória aguda do paciente com DPOC. J Pneumol. 1985; 11(4):185-9.

03. West JB - Fisiologia respiratória. 6a ed. São Paulo: Manole; 2002.
04. Jolliet P, Bulpa P, Chevrolet JC. Effects of the prone position on gas exchange and hemodynamics in severe acute respiratory distress syndrome. Crit Care Med. 1998; 26(12):1977-85. Comment in: Crit Care Med. 1998; 26(12):1934-5.

05. Blanes L, Duarte IS, Calil JA, Ferreira LM. Avaliação clínica e epidemiológica das úlceras por pressão em pacientes internados no Hospital São Paulo. Rev Assoc Med Bras (1992). 2004; 50(2):182-7.

06. Porto EF, Coelho AF. Estudo comparativo da PEEP ide- 
al X PEEP fisiológica em pacientes cardiopatas hemodinamicamente estáveis quando submetidos à Ventilação Mecânica. Rev Soc Cardiol Estado de São Paulo. 2003; 13:(Supl): 114

07. Zanotti E, Rubini F, Iotti G, Braschi A, Palo A, Bruschi C, et al. Elevated static compliance of the total respiratory system: early predictor of weaning unsuccess in severed COPD patients mechanically ventilated. Intensive Care Med. 1995; 21(5):399-405.

08. Auler JO Jr, Carmona MJ, Barbas CV, Saldiva PH, Malbouisson LM. The effects of positive end-expiratory pressure on respiratory system mechanics and hemodynamics in postoperative cardiac surgery patients. Braz J Med Biol Res. 2000; 33(1):31-42.

09. Blanch L, Mancebo J, Perez M, Martinez M, Mas A, Betbese AJ, et al. Short-term effects of prone position in critically ill patients with acute respiratory distress syndrome. Intensive Care Med. 1997; 23(10):1033-9.

10. Gentilello L, Thompson DA, Tonnesen AS, Hernandez D, Kapadia AS, Allen SJ, et al - Effect of a rotating bed on the incidence of pulmonary complications in critically ill patients. Crit Care Med. 1988; 16(8):783-6.

11. Numa AH, Hammer J, Newth CJ. Effect of prone and supine positions on functional residual capacity, oxygenation, and respiratory mechanics in ventilated infants and children. Am J Respir Crit Care Med. 1997; 156(4 Pt
1):1185-9.

12. Kenyon CM, Pedley TJ, Higenbottam TW. Adaptive modeling of the human rib cage in median sternotomy. J Appl Physiol. 1991; 70(5):2287-302.

13. Pelosi P, Bottino N, Chiumello D, Caironi P, Panigada M, Gamberoni C, et al. Sigh in supine and prone position during acute respiratory distress syndrome. Am J Respir Crit Care Med. 2003; 167(4):521-7.

14. Jandre FC, Pino AV, Ascoli A, Giannella-Neto A. Controlador automático da ventilação pulmonary mecânica: projeto e simulação. Rev Bras Eng Biomed. 2002; 18(2):99-110.

15. Servillo G, Roupie E, De Robertis E, Rossano F, Brochard L, Lemaire F, Tufano R. Effects of ventilation in ventral decubitus position on respiratory mechanics in adult respiratory distress syndrome. Intensive Care Med. 1997; 23(12):1219-24.

16. Avanzolini G, Barbini P, Cappello A, Cevenini G. Influence of flow pattern on the parameter estimates of a simple breathing mechanics model. IEEE Trans Biomed Eng. 1995; 42(4):394-402.

17. 17 Manco JC. Fisiologia e fisiopatologia respiratórias. Medicina (Ribeirao Preto). 1998; 31(2):177-90.

18. Green M, Pride NB. Normal respiratory mechanics. In: Scadding JG, Cumming G, Thurlbeck WM. Scientific foundations of respiratory medicine. Philadelphia: W.B. Saunders; 1981. p. 113-29. 